\title{
Upgrade of the Electroporation Device KEA-MOBIL
}

\author{
M. Sack, F. Attmann, R. Stängle, A. Wolf, W. Frey and G. Müller \\ Forschungszentrum Karlsruhe GmbH, Institut für Hochleistungsimpuls- und Mikrowellentechnik \\ Hermann-von-Helmholtz-Platz 1, 76344 Eggenstein-Leopoldshafen, Germany
}

For the production of synthetic bio-fuel the use of green biomass enables additional resources of raw material supplementary to the commonly used dry material. An electroporation assisted drying process gives the opportunity of a more efficient dewatering than only pressing and subsequent drying. Experiments showed a lower relative humidity of the pressed material after electroporation compared to pressing the raw material only. Moreover, the drying process of the electroporated material was much shorter. This is because the pores formed in the cell membranes foster a better extraction of water during pressing and enhance the diffusion process during the drying process. The paper presents exemplarily the experimental results for green rye. For the electroporation-assisted drying process the electric contact to the plant material has to be established by means of the juice inside the plants only rather than adding additional water. Hence, for experiments in larger scale, the existing electroporation device KEA-MOBIL has been equipped with a new combined electroporation reactor and press. The reactor has a plate electrode system, whose upper electrode additionally serves as a piston to apply mechanical force to extract juice for a good electrical contact between plant material and the electrodes. A two-stage Marx generator serves as a pulse source. To reduce the insulation distance to the walls of the shielding cabin the Marx generator is operated in ground symmetric configuration. The device has been designed for a batch-wise throughput of $50 \mathrm{~kg} / \mathrm{h}$. An automatic feeding device enables the alternating use of two test vessels. The device has been tested on-site on a farm with green rye and grass.

PACS numbers: 87.50.cj

\section{Introduction}

Electroporation is a novel method to open the cell membranes of plant cells in industrial scale. In the recent years the feasibility of electroporation devices with a throughput facing the industrial requirements has been shown for example for sugar beets [1], wine grapes and apple mash [2]. During the electroporation process pores in the cell membranes are formed by the application of an electric field to the cells [3]: a charging process enhances the electric field across the membranes, especially in the field direction [4]. After reaching a certain threshold, pores are formed in the membranes. When applying sufficient energy, the pores are opened irreversibly. These pores foster the extraction of substances from biological cells. For an energy-efficient operation pulsed electric fields are applied.

\section{Electroporation-assisted drying process}

Nowadays, with respect to the limited resources of mineral oil, the use of dry and green biomass for the production of fuel is of interest. Green, fresh biomass needs to be dried before it can be used for the gasification process required for the production of synthetic fuel. For a good efficiency of the process an energy saving method for the technical drying process is crucial. In the frame of the BIOLOG-project the efficiency of the processing chain from the growth and harvest of the plants until the production of bio-fuel is investigated. One of the topics of the project is dedicated to the electroporation assisted drying of the plants [5]. The electroporated material enables a larger yield of juice during pressing, because the juice can drain easily through the pores. Hence, evaporation heat for the thermal drying stage is saved.

Figure 1a shows a comparison of the energies for the drying process to $20 \%$ and $0 \%$ relative humidity. Compared to the raw material, approximately half of the evaporation energy can be saved for the electroporated material. The electric energy required for the electroporation has been calculated based on measurements of the current through and the voltage across the electroporation reactor. It is small compared to the thermal energy for drying. Furthermore, a faster decrease of the humidity for the electroporated samples during the thermal drying process due to a better diffusion of water through the electroporated tissue has been observed (Fig. 1b).

Originally, the electroporation device KEA-MOBIL (KEA - Karlsruher Elektroporations Anlage) had been designed for an electroporation in water suspension to guarantee a good electric contact to the electrodes. But for a drying process the use of additional water has to be omitted. Therefore, the electroporation for the purpose of drying is done in the own juice of the plants. Before the application of the pulses, the plants are pressed, until the juice fills the gap between the plant material and 
the electrodes. The pressing force must be kept applied during the pulse application to prevent air from being sucked between the electrodes.

\section{Design of the electroporation reactor and pulse circuit}

For the new electroporation reactor the processing of different sorts of material with a large variety of its characteristics is intended. A batch-wise processing enables a flexible variation of the parameters independent of each other: the control of the pressing force, the pressing time, the electric field strength and the number of applied pulses. The usable volume of the new test vessel has been chosen to $2.5 \mathrm{l}$. It has a cylindrical shape, which has the advantage of an easy manufacturing on a lathe. The upper electrode serves as a piston. To prevent too much pressing force from being led to the walls, the diameter has been chosen wider than the maximum operating distance of the electrodes. A typical conductivity of the pressed plant material has been measured to be $3 \mathrm{mS} / \mathrm{cm}$. With a diameter of $16 \mathrm{~cm}$ and an electrode distance of $12.5 \mathrm{~cm}$ the test vessel has typically a resistance of $20.7 \Omega$. As previous experiments showed, a pulse length of some microseconds at a maximum field of $7 \mathrm{kV} / \mathrm{cm}$ results in a good opening of the cells (Fig. 1). A two-stage Marx generator serves as the pulse source. It is operated in ground-symmetric configuration to keep the device com-

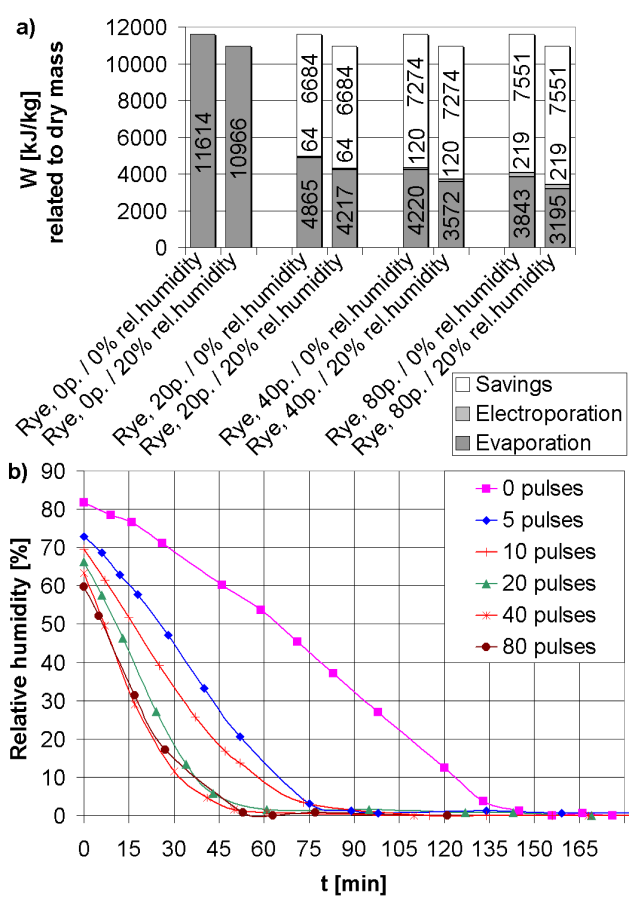

Fig. 1. Energies for drying green rye to $20 \%$ and $0 \%$ relative humidity, (b) temporal decrease of relative humidity during thermal drying at $105^{\circ} \mathrm{C}$, both at $\hat{E}=$ $7 \mathrm{kV} / \mathrm{cm}, t_{h}=1.5 \mu \mathrm{s}$, and depending on the number of applied pulses. pact. With a capacitance of $420 \mathrm{nF}$ per stage according to a calculation a pulse length of $t_{h}=4 \mu \mathrm{s}$ is achieved. The required charging voltage is around $50 \mathrm{kV}$ per stage.

\section{Processing steps and control}

For a throughput of $50 \mathrm{~kg}$ per hour the volume of the test vessel has to be treated every 3 minutes. The treatment consists of the steps: (a) filling of the test vessel, (b) pre-pressing, (c) removing of air bubbles, (d) pulse application, and (e) emptying. For a manual operation, the test setup has to be discharged and grounded safely. Together with the processing steps, this procedure exceeds the time limit of 3 minutes. Therefore, an automated feeding device had to be built.
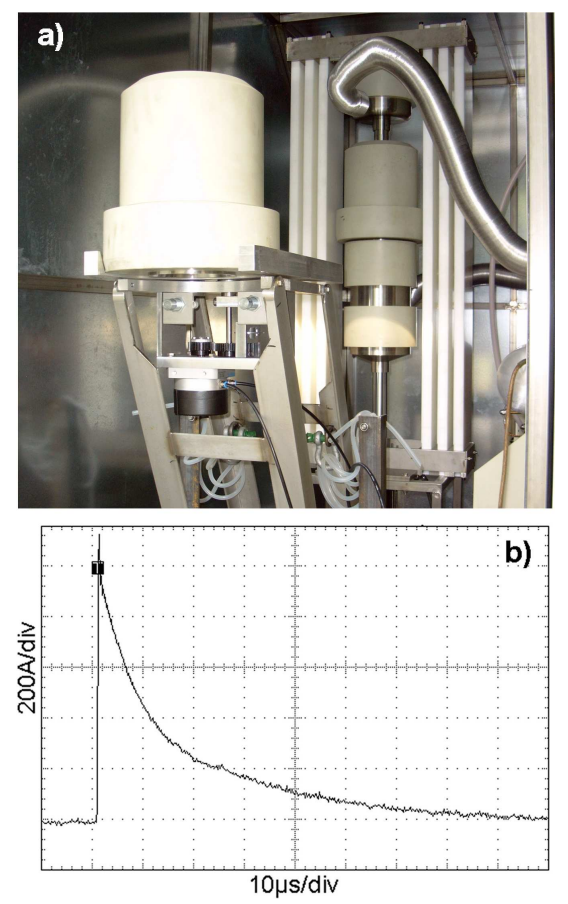

Fig. 2. Press and feeding device inside the shielding cabin. (b) Current shape during the electroporation of grass.

Figure 2a shows a photo of the feeding device in the foreground and the press behind it. In the test setup two test vessels are operated one after the other. The test vessels are filled and emptied manually. They are placed onto a carousel. The test-vessel to be placed into the press next is turned into the direction of the press. A pneumatic cylinder moves it towards the press and a hydraulic piston moves it up towards the fixed stamp to apply the pressing force. For the pulse application the carousel is moved back. Apart from the hydraulic cylinder and the electrodes the main parts of the press are made from insulating material. The press is able to apply a force of $110 \mathrm{kN}$ resulting in a pressure of $54.7 \mathrm{daN} / \mathrm{cm}^{2}$. During the pre-pressing step the air bubbles are removed by a repetitive short decrease of the applied pressure, 
each followed by an increase to its original value. The pulse circuit is placed inside a shielding cabin.

The chain of processing steps is automatically applied by a programmable logic control. Furthermore, the control acquires and displays the relevant process parameters like the ignition voltage of the Marx generator, the charging current and the hydraulic pressure of the press. To omit problems due to electromagnetic interference, most of the measurements are made outside the shielding cabin for the pulse circuit. Signals for the position of the feeding device from inside the cabin are transmitted via fibre optic cables.

\section{First experiments}

The device has been tested successfully on a farm. $50 \mathrm{~kg}$ of grass and $70 \mathrm{~kg}$ of green rye have been treated. The relative humidity of the fresh material was $62 \%$ for green rye and $68 \%$ for grass. Figure $2 \mathrm{~b}$ shows the shape of the measured pulse current for grass. For these experiments the Marx generator has been charged to $21 \mathrm{kV}$ per stage, resulting in $\hat{E}=3.5 \mathrm{kV} / \mathrm{cm}$ across a load resistance of approximately $40 \Omega$. One total processing cycle took approximately $2.2 \mathrm{~min}$. After a subsequent pressing of the electroporated material in a screw extrusion press a relative humidity of $56 \%$ for green rye and $50 \%$ for grass have been obtained.

\section{Conclusions}

The mobile electroporation device KEA-MOBIL has been upgraded for an electroporation in the plant mate- rial's own juice. It has been tested successfully. In some first experiments with green rye and grass a relative humidity of $56 \%$ for green rye and $50 \%$ for grass has been achieved after the final pressing step.

\section{Acknowledgments}

The work was supported by the Bundesministerium für Ernährung, Landwirtschaft und Verbraucherschutz (Federal Ministry of Food, Agriculture, and Consumer Protection of the Federal Republic of Germany) in the frame of the BIOLOG project (Förderkennzeichen 22031405).

\section{References}

[1] M. Sack, C. Schultheiss, H. Bluhm, IEEE Trans. Ind. Appl. 41, 707 (2005).

[2] G. Müller, W. Frey, M. Sack, C. Schultheiss, H.G. Mayer, J. Sigler, M. Kern, U. Günther, Nachr. Forschungszentrum, Vol. 39, 2007.

[3] B. Flaumenbaum, Trudy OTIKP 3, 15 (1949).

[4] K.H. Schoenbach, R.P. Joshi, R.H. Stark, F.C. Dobbs, S.J. Beebe, IEEE Trans. DEI 7, 637 (2000).

[5] M. Sack, C. Eing, T. Berghöfer, L. Buth, R. Stängle, W. Frey, H. Bluhm, IEEE Trans. Plasma Sci. 36, 2577 (2008). 